Preprint: $1-5(1998)$

\title{
NEW INSIGHTS INTO THE EARLY STAGE OF THE GALACTIC CHEMICAL EVOLUTION
}

\author{
Takuji Tsujimoto \\ National Astronomical Observatory, Mitaka, Tokyo, 181-8588 Japan; \\ tsuji@misty.mtk.nao.ac.jp \\ Toshikazu Shigeyama \\ Department of Astronomy, School of Science, University of Tokyo, Bunkyo-ku, Tokyo, 113-0033 Japan \\ Research Center for the Early Universe, School of Science, University of Tokyo, Bunkyo-ku, Tokyo, 113-0033 Japan; \\ shigeyama@astron.s.u-tokyo.ac.jp
}

(Received 10 September 1998; accepted 1 October 1998)

\begin{abstract}
The supernova yields of several heavy elements including $\alpha$-, iron-group, and r-process elements are obtained as a function of the mass of their progenitor main-sequence stars $M_{\mathrm{ms}}$ from the abundance patterns of extremely metal-poor stars with a procedure recently proposed by Shigeyama and Tsujimoto (1998). The ejected masses of $\alpha$ - and iron-group elements increase with $M_{\mathrm{ms}}$, whereas more $\mathrm{Eu}$ is ejected from supernovae with lower $M_{\mathrm{ms}}$. For these several heavy elements, it is shown that the average abundance ratios weighted by the Salpeter initial mass function coincide with the ratios observed in stars with $-2<[\mathrm{Fe} / \mathrm{H}]<-1$ within 0.1 dex. It follows that the correlations of stellar abundance ratios with the metallicity are twofold. One is the abundance ratios for $[\mathrm{Fe} / \mathrm{H}]<-2.5$ imprinted by the nucleosynthesis in individual supernovae on the timescale $\sim 10^{7} \mathrm{yr}$ and the other for $[\mathrm{Fe} / \mathrm{H}]>-2$ results from the mixing of the products from a whole site of the nucleosynthesis, taking place on the timescale longer than $10^{9} \mathrm{yr}$.

Key words: nucleosynthesis - galaxy: evolution — galaxy: solar neighborhood — stars: abundances — stars: Population II - supernovae: general — supernova remnants
\end{abstract}

\section{INTRODUCTION}

Older stars in the Galactic halo show poorer abundances of heavy elements in their spectra if most of heavy elements are well mixed in the halo after they are synthesized in massive stars and ejected by type II supernova (SN II) explosions. Shigeyama \& Tsujimoto (1998) have shown that this age-metallicity relation is broken for extremely metal-poor stars with $[\mathrm{Fe} / \mathrm{H}]<-2.5$ recently observed (McWilliam et al. 1995; Ryan, Norris, \& Beers 1996). Instead their spectra show elemental patterns of individual first generation SNe. This must be a result from that most of the stars with $[\mathrm{Fe} / \mathrm{H}]<-2.5$ were formed from individual supernova remnants (SNRs). It means that utilizing the observed abundance patterns of extremely metal-poor stars, one can construct yields of any observed heavy-element from each SN II.

Shigeyama \& Tsujimoto (1998) have also shown that the mass of Fe ejected by each SN II $\left(M_{\mathrm{Fe}}\right)$ as a function of progenitor mass at the main sequence $\left(M_{\mathrm{ms}}\right)$ can be derived from the observed $[\mathrm{Mg} / \mathrm{Fe}]-[\mathrm{Mg} / \mathrm{H}]$ trend com- bined with the $[\mathrm{Mg} / \mathrm{H}]-M_{\mathrm{ms}}$ relation in theoretical SN models and that this $M_{\mathrm{Fe}}$ as a function of $M_{\mathrm{ms}}$ is consistent with that derived from SN light curve analyses. However this kind of check cannot be applied to the other elements. In this letter, we examine whether the yields of various elements other than Fe obtained by this procedure can reproduce the observed plateau of [element $/ \mathrm{Fe}$ ] seen in the halo stars for $-2<[\mathrm{Fe} / \mathrm{H}]<-1$, which results from a whole population of SNe II integrated over the initial mass function (IMF).

Although the $r$-process nucleosynthesis is poorly understood (Mathews \& Cowan 1990), our scenario can extract information of the site for production from the observed abundances of $r$-process elements on the surface of metal-poor stars. At the same time, it provides a new picture of the chemical evolution for $r$-process elements. Europium is a good indicator of the $r$-process because it is about $90 \%$ of pure $r$-process origin in the Solar System (Howard et al. 1986) and its abundances in stars have been observed by many authors (Butcher 1975; Luck \& Bond 1985; Gilroy et al. 1988; Magain 1989; Woolf, Tomkin, \& Lambert 1995 McWilliam et al. 
1995; Ryan et al. 1996). The observed trend of [Eu/Fe]$[\mathrm{Fe} / \mathrm{H}]$ for $[\mathrm{Fe} / \mathrm{H}]<-2$ before McWilliam et al. (1995) and Ryan et al. (1996) was regarded as the decrease in the $[\mathrm{Eu} / \mathrm{Fe}]$ ratio as $[\mathrm{Fe} / \mathrm{H}]$ decreases, which was ascribed to the later production of the $r$-process element than that of iron; The $r$-process occurs in low-mass SNe II with initial masses of $10-11 M_{\odot}$ (Mathews \& Cowan 1990; Mathews, Bazan, \& Cowan 1992). The observations (McWilliam et al. 1995; Ryan et al. 1996) covering stars with $[\mathrm{Fe} / \mathrm{H}] \sim-4$ have drastically changed the observed trend of $[\mathrm{Eu} / \mathrm{Fe}]-[\mathrm{Fe} / \mathrm{H}]$ for $[\mathrm{Fe} / \mathrm{H}]<-2$, indicating the increase in the $[\mathrm{Eu} / \mathrm{Fe}]$ ratio as $[\mathrm{Fe} / \mathrm{H}]$ decreases. Such an observed trend is incompatible with the possible $r$-process sites proposed so far, whereas from our point of view, the observed trend in the range of $[\mathrm{Fe} / \mathrm{H}]<-2.5$ reflects different $[\mathrm{Eu} / \mathrm{Fe}]$ ratios in SNRs with different progenitor masses.

In this letter, we will show that the heavy-element yields for $\alpha$-, iron-group elements and $\mathrm{Eu}$ derived from the analysis of abundance pattern in extremely metalpoor stars are quite reliable ones and that combining yields thus obtained with the Galactic chemical evolution model, the observed abundance patterns over the whole metallicity range can be well reproduced.

\section{WELL-MIXED OR INHOMOGENEOUS?}

The yields of heavy elements not influenced by the mass cut between the forming neutron star (or black hole) and the ejected envelope are expected to be more reliably calculated in SN II models than those influenced by the mass cut. Therefore the observed abundance ratios of $[\mathrm{C} / \mathrm{Mg}]$ with respect to $[\mathrm{Mg} / \mathrm{H}]$ can tell whether extremely metalpoor stars were formed from individual SNe or from the well-mixed interstellar gas. The observed correlation of [C/Mg] with $[\mathrm{Mg} / \mathrm{H}]$ (McWilliam et al. 1995) is shown in Figure 1. The dashed curve in the same figure is the correlation of these ratios predicted from the one-zone Galactic chemical evolution model in which the gas is distributed uniformly and the heavy elements are wellmixed within the zone. Here we use the updated nucleosynthesis calculations by Nomoto et al. (1997) for SN II yields and the results of van den Hoek \& Groenewegen (1997) for the AGB star's yields. Details of the basic model ingredients are presented in Yoshii, Tsujimoto, \& Kawara (1998). This obvious disagreement with the observation indicates that the mixing of elements did not take place at that time.

The open and filled circles are the correlation of the abundance ratios in SNRs at the end of expansions calculated from two theoretical SN models (Nomoto et al. 1997; Woosley \& Weaver 1995). The coincidence of the two correlations must be a result from that most of the stars with $[\mathrm{Mg} / \mathrm{H}]<-2$ were formed from individual

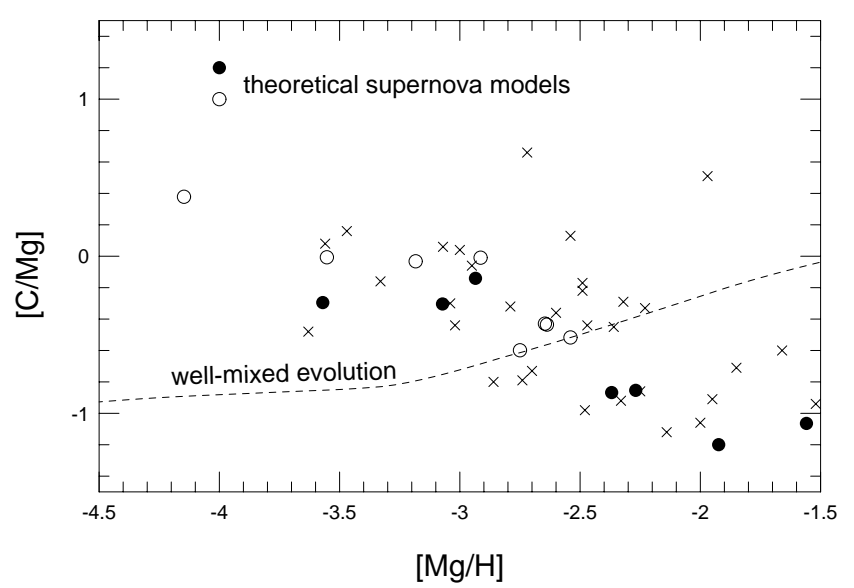

Fig. 1. Comparison of the observed correlation of $[\mathrm{C} / \mathrm{Mg}]-[\mathrm{Mg} / \mathrm{H}]$ (crosses: McWilliam et al. 1995) with the theoretical nucleosynthesis $[\mathrm{C} / \mathrm{Mg}]$ ratio from type II supernovae as a function of the predicted $[\mathrm{Mg} / \mathrm{H}]$ (filled circles: Nomoto et al. 1997; open circles: Woosley \& Weaver 1995). The evolutionary change in $[\mathrm{C} / \mathrm{Mg}]$ predicted by the onezone chemical evolution model including the contribution from AGB stars (van den Hoek \& Groenewegen 1997) is also shown by the dashed curve.

SNRs. Therefore the observed correlation must be made within the first $\sim 2 \times 10^{7} \mathrm{yr}$ (corresponding to the life time of the least massive star that explodes as a SN II) from the birth of the first generation stars. During this period, a SNR scarcely intersects with another SNR (Audouze \& Silk 1995).

\section{THE IMF-WEIGHTED ABUNDANCE RA- TIOS}

The observed correlations of abundance ratios in halo stars with higher $[\mathrm{Fe} / \mathrm{H}]([\mathrm{Mg} / \mathrm{H}])$ result from the mixing of elements in the Galactic halo. How precise the abundance pattern of stars for the range of $-4<[\mathrm{Fe} / \mathrm{H}]<-2.5$ represents the nucleosynthesis yield of each supernova can be checked by the comparison of their average nucleosynthesis yield with the observed plateau (e.g., Gilroy et al. 1988; Gratton 1989; Magain 1989; Gratton \& Sneden 1991; Nissen et al. 1994) seen in the [element/Fe] ratio for $-2<[\mathrm{Fe} / \mathrm{H}]<-1$, which results from a whole population of SNe II integrated over the IMF. Using the same method as applied to the iron yield, we can obtain the supernova yields for $\alpha$ - and iron-group elements as a function of the initial stellar mass. The procedure is as follows;

1. For each $\mathrm{SN}$, the $[\mathrm{Mg} / \mathrm{H}]$ ratio is calculated from the ratio of the mass of $\mathrm{Mg}$ ejected from a SN II to the 
mass of hydrogen swept up by the SNR (Shigeyama \& Tsujimoto 1998) and thus the theoretical $[\mathrm{Mg} / \mathrm{H}]-$ $M_{\mathrm{ms}}$ relation is derived.

2. We perform the $\chi^{2}$ fit of the observational data in the $[\mathrm{Mg} /$ element $]-[\mathrm{Mg} / \mathrm{H}]$ diagram.

3. We combine this curve with the theoretical $[\mathrm{Mg} / \mathrm{H}]$ $M_{\mathrm{ms}}$ relation and obtain the $[\mathrm{Mg} /$ element $]$ ratio for each $M_{\mathrm{ms}}$.

4. Using the theoretical nucleosynthesis mass of $\mathrm{Mg}$ for each $M_{\mathrm{ms}}$, the ejected mass of element as a function of $M_{\mathrm{ms}}$ is derived.

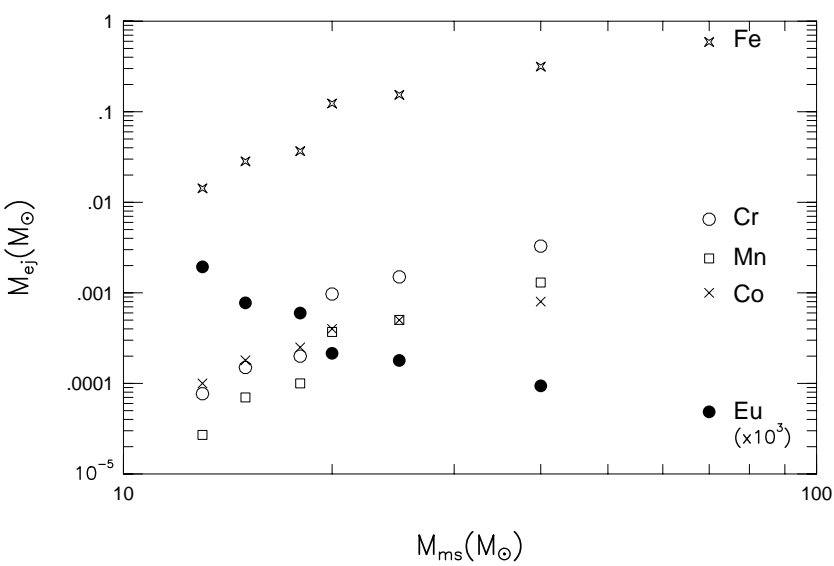

Fig. 2. Ejected masses of heavy elements from $\mathrm{SNe}$ II as a function of the mass of their progenitor main-sequence stars. They are derived from the observed abundance pattern in extremely metalpoor stars combined with the $[\mathrm{Mg} / \mathrm{H}]-M_{\mathrm{ms}}$ relation in the theoretical SN models.

The yields thus obtained are shown for several elements in Figure 2. Here we use the theoretical supernova model by Nomoto et al. (1997). The iron-group elements are produced in the deepest layers of the ejecta. Therefore a slightly different stellar-mass dependence of the Co yield from other iron-group elements may indicate how the explosion separates material comprising the neutron star from that of the ejecta. We then calculate the average nucleosynthesis yields integrated over $10 M_{\odot}<M_{\mathrm{ms}}<50 M_{\odot}$ assuming the Salpeter IMF, and obtain the average [element/Fe] ratios. They are tabulated with the observed values in Table 1 . The predicted average abundance ratios are all consistent with the observed ones within an error of $\sim 0.1$ dex. This good agreement strongly supports the rightness of our determination of each supernova yield and suggests that the observed correlations of stellar abundance ratios with the metallicity are twofold: the nucleosynthesis abundance ratios for $[\mathrm{Fe} / \mathrm{H}]<-2.5$, which in each star are imprinted by the nucleosynthesis of a single supernova and the average abundance ratios for $[\mathrm{Fe} / \mathrm{H}]>-2$, which result from a whole site for production.

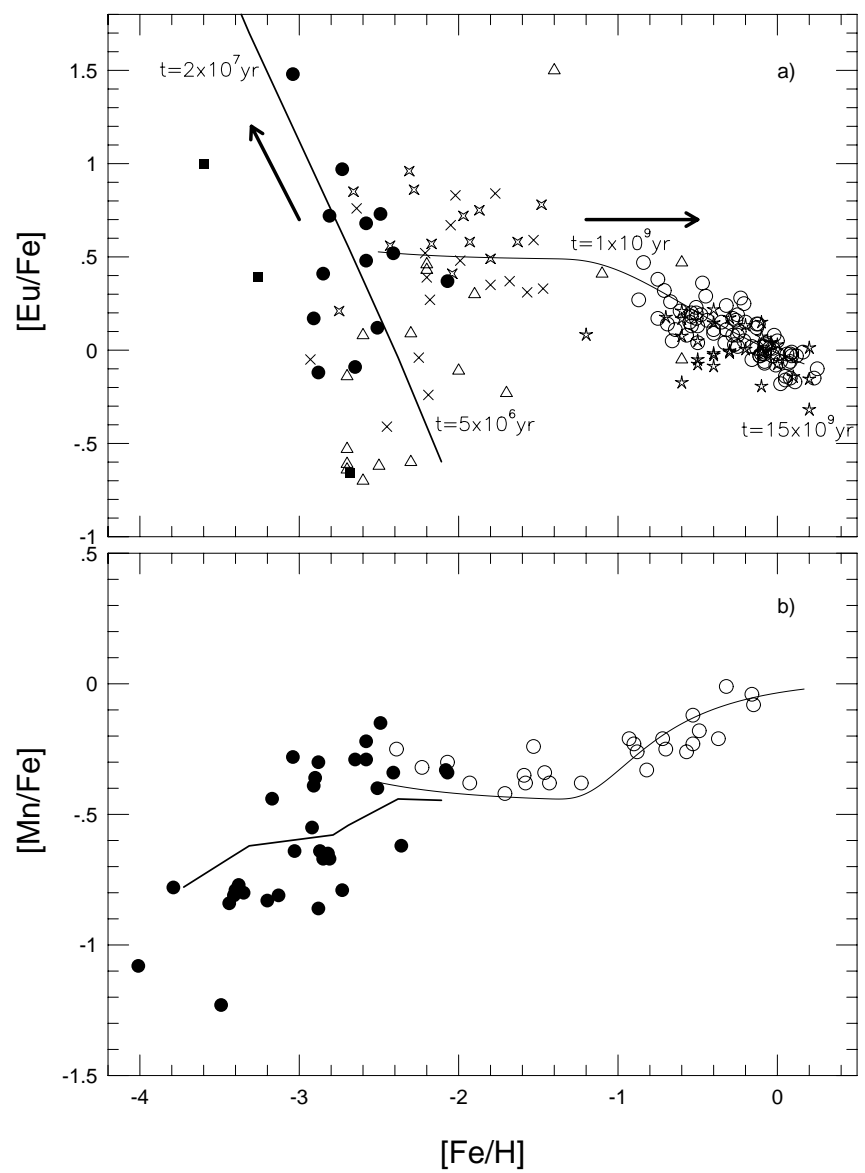

Fig. 3. (a) Correlation of $[\mathrm{Eu} / \mathrm{Fe}]$ with $[\mathrm{Fe} / \mathrm{H}]$. The symbols represent the data taken from various refs (filled circles: McWilliam et al. 1995; filled squares: Ryan et al. 1996; open triangles: Luck \& Bond 1985; four-pointed stars: Gilroy et al. 1988; crosses: Magain 1989; open circles: Woolf et al. 1995; five-pointed stars: Butcher et al. 1975). See text for details. (b) the same as (a) but for $[\mathrm{Mn} / \mathrm{Fe}]$. The circles are data from McWilliam et al. (1995) (filled circles) and Gratton (1989) (open circles). See text for details.

It is noted that the present nucleosynthesis models (Nomoto et al. 1997; Woosley \& Weaver 1995) cannot reproduce the observed plateau values for most of the elements in Table 1 due to a problem in modelling a SN II explosion or mass cut (Thielemann, Hashimoto, \& Nomoto 1990). There are indications from Galactic evolution models (Timmes, Woosley, \& Weaver 1995; Samland 1998) that the nucleosynthesis models overestimate the Fe yields by a factor of two. However our results give almost the same average Fe yield as those predicted by the nucleosynthesis models and suggest that the heavy- 
Table 1. The comparison of the predicted abundance ratios of the halo stars in the range of $-2<[\mathrm{Fe} / \mathrm{H}]<-1$ with the observations

\begin{tabular}{ccccccc}
\hline \hline & prediction & \multicolumn{5}{c}{ observation } \\
\cline { 3 - 6 }$[\mathrm{ele} / \mathrm{Fe}]$ & & Nissen(94) & Gratton\&Sneden(91) & Gratton(89) & Magain(89) & Gilroy $(88)$ \\
\hline$[\mathrm{Mg} / \mathrm{Fe}]$ & +0.43 & $+0.41 \pm 0.07$ & - & - & $+0.47 \pm 0.09$ & - \\
{$[\mathrm{Ca} / \mathrm{Fe}]$} & +0.44 & $+0.35 \pm 0.06$ & $+0.29 \pm 0.06$ & - & $+0.47 \pm 0.08$ & $+0.25 \pm 0.20$ \\
{$[\mathrm{Cr} / \mathrm{Fe}]$} & -0.19 & $-0.11 \pm 0.07$ & $-0.04 \pm 0.05$ & - & $+0.01 \pm 0.08$ & $-0.12 \pm 0.24$ \\
{$[\mathrm{Mn} / \mathrm{Fe}]$} & -0.42 & - & $-0.31 \pm 0.07$ & $-0.34 \pm 0.06$ & - & - \\
{$[\mathrm{Co} / \mathrm{Fe}]$} & +0.02 & - & $-0.12 \pm 0.05$ & - & - & $-0.37 \pm 0.34$ \\
{$[\mathrm{Ti} / \mathrm{Fe}]$} & +0.36 & $+0.27 \pm 0.06$ & $+0.28 \pm 0.10$ & - & $+0.40 \pm 0.09$ & $+0.08 \pm 0.10$ \\
{$[\mathrm{Ni} / \mathrm{Fe}]$} & +0.09 & - & $-0.04 \pm 0.08$ & - & - & $-0.08 \pm 0.19$ \\
\hline
\end{tabular}

element yields predicted by them should be revised except for a few elements such as $\mathrm{C}, \mathrm{Mg}$ and $\mathrm{O}$.

\section{4. $R$-PROCESS ELEMENT}

Figure $3 \mathrm{a}$ shows the observed correlation of $[\mathrm{Eu} / \mathrm{Fe}]$ as a function of $[\mathrm{Fe} / \mathrm{H}]$. The filled symbols (McWilliam et al. 1995; Ryan et al. 1996) indicate the increase in the $[\mathrm{Eu} / \mathrm{Fe}]$ ratio as $[\mathrm{Fe} / \mathrm{H}]$ decreases for the range of $[\mathrm{Fe} / \mathrm{H}]$ $<-2$, which can be ascribed to different $[\mathrm{Eu} / \mathrm{Fe}]$ ratios in SNRs with different progenitor masses.

The ejected mass of Eu as a function of the initial stellar mass can be obtained by the same method as for $\alpha$ and iron-group elements (see $\S 3$ ), i.e., derived from the observed [Eu/Mg]-[Mg/H] trend (McWilliam et al. 1995) combined with the $[\mathrm{Mg} / \mathrm{H}]-M_{\mathrm{ms}}$ relation in the theoretical SN models, and is shown in Figure 2. What's interesting is that $\mathrm{Eu}$ is ejected more in a less massive star, different from the cases for other elements. This implies that $\mathrm{Eu}$ is produced more in less massive stars or that more Eu eventually falls back onto the nascent neutron stars in massive SNe II. The thick line in Figure 3a indicates the predicted nucleosynthesis $[\mathrm{Eu} / \mathrm{Fe}]$ ratios in $18-50 M_{\odot}$ stars. The lower mass limit corresponds to the most metal-deficient star observed by McWilliam et al. (1995). As a more massive star's explosion corresponds to a higher $[\mathrm{Fe} / \mathrm{H}]$, the time elapses toward lower metallicity as indicated by the arrow for $[\mathrm{Fe} / \mathrm{H}]<-2.5$ in Figure 3a.

Using the nucleosynthesis yields of $\mathrm{Fe}$ and $\mathrm{Eu}$ inferred from extremely metal-poor stars, we calculate the time evolution of $[\mathrm{Eu} / \mathrm{Fe}]$ with the one-zone model of Galactic chemical evolution (Yoshii et al. 1998) and the result is shown in the $[\mathrm{Eu} / \mathrm{Fe}]-[\mathrm{Fe} / \mathrm{H}]$ diagram for $[\mathrm{Fe} / \mathrm{H}]>-2.5$ (the thin line in Figure $3 \mathrm{a}$ ). The predicted plateau in $-2<[\mathrm{Fe} / \mathrm{H}]<-1$ is consistent with the observed ratios. The decrease in $[\mathrm{Eu} / \mathrm{Fe}]$ as $[\mathrm{Fe} / \mathrm{H}]$ increases from -1 to 0 is caused by the iron production from type Ia supernovae, which eject their iron $\sim 1 \mathrm{Gyr}$ later. The sequence of the predicted nucleosynthesis $[\mathrm{Eu} / \mathrm{Fe}]$ ratio for $[\mathrm{Fe} / \mathrm{H}]$ $\lesssim-2$ (thick line) does not connect smoothly with the $[\mathrm{Eu} / \mathrm{Fe}]$ plateau. It is a consequence of the decreasing mass of Eu with the increasing stellar mass as shown in Figure 2, which leads to the relatively small contribution from the massive SNe II to the IMF-averaged value. This gap might yield the large scatter in $[\mathrm{Eu} / \mathrm{Fe}]$ until the $[\mathrm{Eu} / \mathrm{Fe}]$ ratios converge to the average plateau ratio. As shown by the two solid lines in Figure 3a, the observed abundance ratios are created in two different enrichment epochs with different time scales; the sequence of the first SN II explosions and the phase approximated by the well-mixed evolution over several Gyrs in which the age-metallicity relation holds. For comparison, we show that the evolutionary change in $[\mathrm{Mn} / \mathrm{Fe}]$ against $[\mathrm{Fe} / \mathrm{H}]$ is also composed of two different phases (Figure $3 \mathrm{~b})$. The $[\mathrm{Mn} / \mathrm{Fe}]$ ratio from individual $\mathrm{SNe}$ II at $[\mathrm{Fe} / \mathrm{H}]$ $\sim-2.5$ connects with the $[\mathrm{Mn} / \mathrm{Fe}]$ plateau, because the IMF-averaged $[\mathrm{Mn} / \mathrm{Fe}]$ ratio is similar to the nucleosynthesis $[\mathrm{Mn} / \mathrm{Fe}]$ ratio of the most massive star that ejects about two orders of magnitude larger mass of Mn than the lower mass SN II (see Figure 2).

\section{CONCLUSION}

We conclude that each extremely metal-poor star with $[\mathrm{Fe} / \mathrm{H}]<-2.5$ was formed from a single supernova event and in this early stage the age-metallicity relation does not hold, but the time elapses toward lower metallicity. Thus the observed abundance pattern of these stars which reflect the first generation supernova ejecta enables us to determine the heavy-element yields ejected from SNe II for any observed element as a function of the initial stellar mass. It is noted that we determine the yields descending from the metal-free progenitor stars. However the metallicity effect on the yields cannot be seen in the present study such as (i) the Fe yield consistent with that derived from SN light curve analyses and (ii) the observed $[\mathrm{C} / \mathrm{Mg}]$ trend that can be reproduced by the SN model in which the initial metallicity is solar.

The accuracy of our determination is guaranteed by the quite good agreement of the IMF-weight ed abundance ratios with the observed plateau seen in the halo stars for $-2<[\mathrm{Fe} / \mathrm{H}]<-1$. The yields thus obtained are indispensable because theoretical SN II models to 
date can reliably predict the amounts of only a few elements. Progress in observations of not only the abundance pattern in extremely metal-poor stars including the oxygen abundance but also the constant abundance ratios in metal-poor halo stars promises the acquisition of more reliable yields for SNe.

As the most intriguing example, we have demonstrated that $\mathrm{Eu}$ is ejected more in less massive stars. This mass dependence is reverse of those for iron-group elements. We predict that such a difference in the mass dependence of the ejected mass is (at least partly) responsible for the difference in the scatter of the observed abundance ratios.

Combining the yields obtained by our procedure with the Galactic chemical evolution model, we have shown that the observed abundance patterns over the whole metallicity range can be well reproduced, where we introduce two different enrichment phases with different time scales, e.g., the sequence of the first SN II explosions within $\sim 2 \times 10^{7}$ yr and the well-mixed phase over several Gyrs. The transition between them will be discussed in the forthcoming paper.

This work has been partially supported by COE research (07CE2002) of the Ministry of Education, Science, Culture, and Sports in Japan.

\section{References}

Audouze, J., \& Silk, J. 1995, ApJ, 451, L49

Butcher, H. R. 1985, ApJ, 199, 710

Gilroy, K. K., Sneden, C., Pilachowski, C. A., \& Cowan, J. J. 1988, ApJ, 327, 298

Gratton, R. G., \& Sneden, C. 1991, A\&A, 241, 501

Gratton, R. G. 1989, A\&A, 208, 171

Luck, R. E., \& Bond, H. E. 1985, ApJ, 292, 559

Magain, P. 1989, A\&A, 209, 211

Mathews, G. J., \& Cowan, J. J. 1990, Nature, 345, 491

Mathews, G. J., Bazan, G., \& Cowan, J. J. 1992, ApJ, 391,719

McWilliam, A., Preston, G. W., Sneden, C., \& Searle, L. 1995, AJ, 109, 2757

Nissen, P. E., Gustafsson, B., Edvardsson, B., \& Gilmore, G. 1994, A\&A, 285, 440

Nomoto, K., Hashimoto, M., Tsujimoto, T., Thielemann, F.-K., Kishimoto, N., Kubo, Y., \& Nakasato, N. 1997, Nuclear Physics A, 616, 79c

Ryan, S. G., Norris, J. E., \& Beers, T. C. 1996, ApJ, 471, 254

Samland, M. 1998, ApJ, 496, 155

Shigeyama, T., \& Tsujimoto, T. 1998, ApJ, in press

Timmes, F. X., Woosley, S. E., \& Weaver, T. A. 1995, ApJS, 98, 617

van den Hoek, L. B., \& Groenewegen, M. A. T. 1997, A\&AS, 123, 305
Yoshii, Y., Tsujimoto, T. \& Kawara, K. 1998, ApJ, in press

Woolf, V. M., Tomkin, J., \& Lambert, D. 1995, ApJ, 453, 660

Woosley, S. E., \& Weaver, T. A. 1995, ApJS, 101, 181 